\title{
Smoluchowski approach to nonlinear Vlasov-Fokker-Planck equations: Stability analysis of beam dynamics and Haïssinski theory
}

\author{
T. D. Frank \\ Institute for Theoretical Physics, University of Münster, Wilhelm-Klemm-Straße 9, 48149 Münster, Germany
}

(Received 13 April 2006; published 14 August 2006)

\begin{abstract}
For a class of Vlasov-Fokker-Planck equations that have frequently been used to examine beam dynamic instabilities in accelerators and storage rings, it is shown that the stability of stationary distributions can be determined by studying reduced models defined by Smoluchowski equations. This is illustrated explicitly for longitudinal particle bunches in beams subjected to a particular class of wake fields, described by a Haïssinski distribution. For this class we find that continuous Haïssinski distributions are stable because they correspond to minima of appropriately defined free energy functionals. For parameters for which continuous distributions no longer exist, discontinuous distributions may still exist but correspond to free energy maxima and saddle points and are unstable.
\end{abstract}

DOI: 10.1103/PhysRevSTAB.9.084401

PACS numbers: 29.27.Bd, 41.75.-i, 52.25.Dg

\section{INTRODUCTION}

Nonlinear Vlasov-Fokker-Planck equations have frequently been used to describe beam dynamics in accelerators and storage rings. On account of their nonlinearity, they are helpful approaches to examine beam dynamic instabilities [1-6]. Nonlinear Vlasov-Fokker-Planck equations that describe beam dynamic instabilities typically involve two different kinds of state variables such as particle positions and energies. In general, the stability of such nonlinear models has to be examined on the complete phase spaces spanned by these variables. However, in accelerator physics we are often dealing with mean field interactions (e.g. interactions due to wake fields or particleparticle interactions) that involve only particle positions. Therefore, the question arises whether or not the stability of accelerator beams can be examined in the subspace describing particle positions. That is, can we determine the stability of particle beams by means of reduced dynamical models? If this question can be answered in the affirmative, analytical and numerical methods for the stability analysis of beam dynamics could in general be considerably simplified.

In the present study it will be shown that for a fundamental class of nonlinear Vlasov-Fokker-Planck equations it is sufficient to perform stability analysis on reduced dynamical models defined by Smoluchowski equations. In order to demonstrate this point, we will first view position and energy variables as counterparts of position and momentum variables of particles belonging to selfinteracting Hamiltonian systems. We will show that the stability of such Hamiltonian systems can be determined by studying appropriately defined Smoluchowski equations (see Sec. II A). In this context, we will confine ourselves to studying self-interacting Hamiltonian functions with symmetric kernels. This implies that we will consider only accelerator systems with odd wake functions (i.e. with purely imaginary impedances) [7], Sec. 2.3. The Smoluchowski approach will then be applied to examine the stability of particle bunches in longitudinal beams as predicted by the Haiissinski theory (see Sec. II B). That is, we will consider a wake field function given by the derivative of the Dirac delta distribution. In this context, it will also be shown that the Smoluchowski approach is consistent with alternative approaches reported in earlier studies $[8,9]$.

\section{SMOLUCHOWSKI APPROACH}

In order to emphasize the generality of our approach, we consider first a wide class of self-interacting Hamiltonian systems described by nonlinear Vlasov-Fokker-Planck equations such as can be found in plasma physics, astrophysics as well as accelerator physics. Accordingly, let $\mathbf{p}, \mathbf{q}$ denote generalized momentum and position variables of such Hamiltonian systems. Let $P(\mathbf{p}, \mathbf{q}, t)=\langle\delta[\mathbf{q}-$ $\mathbf{q}(t)] \delta[\mathbf{p}-\mathbf{p}(t)]\rangle$ describe the probability density (distribution function) of $\mathbf{p}$ and $\mathbf{q}$, where $\delta(\cdot)$ denotes the Dirac delta function and averages $\langle\cdot\rangle$ refer to ensemble averages. Likewise, let $\rho(\mathbf{q}, t)$ and $\sigma(\mathbf{p}, t)$ denote the reduced probability densities $\rho(\mathbf{q}, t)=\int P(\mathbf{p}, \mathbf{q}, t) \mathrm{d}^{3} p$ and $\sigma(\mathbf{p}, t)=$ $\int P(\mathbf{p}, \mathbf{q}, t) \mathrm{d}^{3} q$, respectively. We will consider systems exhibiting Hamiltonian functions of the form

$$
H(\mathbf{p}, \mathbf{q})=\frac{p^{2}}{2 m}+V[\mathbf{q}, \rho]
$$

with $p^{2}=|\mathbf{p}|^{2}$, where $V$ is a self-consistent potential that depends on the reduced probability density $\rho$. We assume that the potential $V$ is composed of a single particle potential $V_{0}$ and a mean field interaction potential $G$ such that we can write

$$
V[\mathbf{q}, \rho(\mathbf{q}, t)]=V_{0}(\mathbf{q})+\int G\left(\mathbf{q}, \mathbf{q}^{\prime}\right) \rho\left(\mathbf{q}^{\prime}, t\right) \mathrm{d} q^{13} .
$$


The function $G$ is assumed to be symmetric: $G\left(\mathbf{q}, \mathbf{q}^{\prime}\right)=$ $G\left(\mathbf{q}^{\prime}, \mathbf{q}\right)$. In the context of beam dynamics, $G\left(\mathbf{q}, \mathbf{q}^{\prime}\right)=$ $G\left(\mathbf{q}-\mathbf{q}^{\prime}\right)$ corresponds to an integrated wake field function like $G(\mathbf{z})=\int^{\mathbf{z}} W\left(\mathbf{z}^{\prime}\right) \mathrm{d} \mathbf{z}^{\prime}$, where $W$ denotes the wake field. The symmetry constraint on $G$ then implies that $W$ must be an odd function.

We will account for dissipation and fluctuations using the framework of canonical-dissipative systems [10-16] such that the stochastic Hamiltonian dynamics finally reads

$$
\frac{\mathrm{d}}{\mathrm{d} t} \mathbf{q}=\frac{\partial}{\partial \mathbf{p}} H \quad \frac{\mathrm{d}}{\mathrm{d} t} \mathbf{p}=-\frac{\partial}{\partial \mathbf{q}} H-\frac{\gamma}{m} \mathbf{p}+\sqrt{\gamma k_{B} T} \boldsymbol{\Gamma}(t),
$$

where $\boldsymbol{\Gamma}$ is a Langevin force [17] normalized with respect to the Dirac delta function like $\left\langle\Gamma_{i}(t) \Gamma_{k}\left(t^{\prime}\right)\right\rangle=2 \delta_{i k} \delta(t-$ $\left.t^{\prime}\right)$ with $i, k=1,2,3$. Here, $T$ is regarded as an equilibrium temperature and $k_{B}$ denotes the Boltzmann constant. In Sec. II B we will reinterpret the product of these two parameters simply as a diffusion constant or a noise amplitude. The nonlinear Vlasov-Fokker-Planck equation corresponding to Eq. (3) reads ([18], Sec. 4.6)

$$
\begin{aligned}
\frac{\partial}{\partial t} P(\mathbf{p}, \mathbf{q}, t)= & -\frac{\partial}{\partial \mathbf{q}}\left(\frac{\partial H}{\partial \mathbf{p}} P\right)+\frac{\partial}{\partial \mathbf{p}}\left(\frac{\partial H}{\partial \mathbf{q}} P\right) \\
& +\gamma \frac{\partial}{\partial \mathbf{p}}\left[\left(\frac{\mathbf{p}}{m}+k_{B} T \frac{\partial}{\partial \mathbf{p}}\right) P\right]
\end{aligned}
$$

The stationary distribution $P_{\mathrm{st}}$ factorizes like $P_{\mathrm{st}}(\mathbf{p}, \mathbf{q})=$ $\sigma_{\text {st }}(\mathbf{p}) \rho_{\text {st }}(\mathbf{q})$ and its kinetic part is given by the MaxwellBoltzmann distribution

$$
\sigma_{\mathrm{st}}(\mathbf{p})=\left(\frac{m}{2 \pi k_{B} T}\right)^{3 / 2} \exp \left\{-\frac{p^{2}}{2 m k_{B} T}\right\}
$$

The distribution $\rho_{\mathrm{st}}(\mathbf{q})$ is implicitly defined by means of the self-consistency equation

$$
\rho_{\mathrm{st}}(\mathbf{q})=\frac{1}{Z} \exp \left\{-\frac{V\left[\mathbf{q}, \rho_{\mathrm{st}}(\mathbf{q})\right]}{k_{B} T}\right\}
$$

where $Z$ denotes a normalization constant.

\section{A. Stability analysis}

In what follows we will exploit the stability analysis by means of Lyapunov's direct method and the free energy approach to nonlinear Fokker-Planck equations as proposed by Shiino [19,20] and reviewed in [18], Sec. 5.1.

\section{Lyapunov's direct method: A simple example}

For the following discussion it will be helpful to recall the basic steps of Lyapunov's direct method. To this end, we consider the simple example of a first-order dynamical system given by $\mathrm{d} x(t) / \mathrm{d} t=-\mathrm{d} L(x) / \mathrm{d} x$, where $x(t)$ is a time-dependent state variable and $L(x)$ a potential function. In this case, it is clear that

(i) the potential $L$ is a monotonically decreasing function with respect to $t$ for solutions $x(t)$ (because we have $\left.\mathrm{d} L / \mathrm{d} t=-|\mathrm{d} L / \mathrm{d} x|^{2}\right)$;

(ii) fixed points $x_{\mathrm{st}}$ correspond to extrema of $L$ (i.e. we have $\mathrm{d} x / \mathrm{d} t=0 \Leftrightarrow \mathrm{d} L / \mathrm{d} x=0)$;

(iii) if $L$ is bounded from below then from the two aforementioned properties it follows that $L$ becomes stationary in the limit $t \rightarrow \infty$ which in turn implies that $x(t)$ converges to a fixed point $x_{\mathrm{st}}$;

(iv) irrespective of the boundedness of $L$, it follows that a fixed point $x_{\text {st }}$ is stable (unstable) if the inequality $\mathrm{d}^{2} L / \mathrm{d} x^{2}>0\left(\mathrm{~d}^{2} L / \mathrm{d} x^{2}<0\right)$ holds at $x=x_{\mathrm{st}}$.

\section{Free energy approach in full phase space}

Next, we will apply Lyapunov's direct method to the Vlasov-Fokker-Planck equation (4). To this end, we will replace the aforementioned quantities $x(t)$ and $L(x)$ by the probability density $P(\mathbf{p}, \mathbf{q}, t)$ and a free energy functional $F$ defined on $P$. Let $U$ denote the internal energy of the Hamiltonian system (1) given by $U=\left\langle p^{2} /(2 m)+\right.$ $\left.V_{0}(\mathbf{q})\right\rangle+0.5 \int G\left(\mathbf{q}, \mathbf{q}^{\prime}\right) \rho(\mathbf{q}, t) \rho\left(\mathbf{q}^{\prime}, t\right) \mathrm{d} q^{3} \mathrm{~d} q^{\prime 3}$. Let $S$ describe the Boltzmann-Gibbs-Shannon entropy $S=$ $-k_{B} \int P \ln P \mathrm{~d} p^{3} \mathrm{~d} q^{3}$. Then, the free energy $F$ of the Hamiltonian system (1) is given by $F=U-T S$. That is, we have

$$
\begin{aligned}
F= & \left\langle\frac{p^{2}}{2 m}+V_{0}(\mathbf{q})\right\rangle+\frac{1}{2} \int G\left(\mathbf{q}, \mathbf{q}^{\prime}\right) \rho(\mathbf{q}, t) \rho\left(\mathbf{q}^{\prime}, t\right) \mathrm{d} q^{3} \mathrm{~d} q^{\prime 3} \\
& +k_{B} T \int P \ln P \mathrm{~d} p^{3} \mathrm{~d} q^{3} .
\end{aligned}
$$

Note that the variational derivative of $U$ with respect to $P$ is given by $\delta U / \delta P=H$. Likewise, we have

$$
\begin{aligned}
\frac{\delta F}{\delta P}= & \frac{p^{2}}{2 m}+V_{0}(\mathbf{q})+\int G\left(\mathbf{q}, \mathbf{q}^{\prime}\right) \rho\left(\mathbf{q}^{\prime}, t\right) \mathrm{d} q^{\prime 3} \\
& +k_{B} T(1+\ln P) .
\end{aligned}
$$

Let us define the gradient $\nabla=(\partial / \partial \mathbf{p}, \partial / \partial \mathbf{q})$ of the sixdimensional phase space spanned by $\mathbf{p}$ and $\mathbf{q}$. Using the reversible drift $\mathbf{I}=\left(\mathbf{I}_{p}, \mathbf{I}_{q}\right)=(-\partial H / \partial \mathbf{q}, \partial H / \partial \mathbf{p})$ and Eq. (8), the Vlasov-Fokker-Planck equation (4) can be written as

$$
\frac{\partial}{\partial t} P(\mathbf{p}, \mathbf{q}, t)=-\nabla \cdot(\mathbf{I} P)+\gamma \frac{\partial}{\partial \mathbf{p}}\left(P \frac{\partial}{\partial \mathbf{p}} \frac{\delta F}{\delta P}\right)
$$

(see also [18,21]). It can be shown that for solutions $P(\mathbf{p}, \mathbf{q}, t)$ of Eq. (9) the free energy $F$ as a function of time evolves like

$$
\frac{\mathrm{d}}{\mathrm{d} t} F=-\left\langle\mathbf{X}^{\text {th }} \cdot \mathbf{I}\right\rangle-\gamma \int P\left|\frac{\partial}{\partial \mathbf{p}} \frac{\delta F}{\delta P}\right|^{2} \mathrm{~d} p^{3} \mathrm{~d} q^{3}
$$

with $\mathbf{X}^{\text {th }}=-\nabla \delta F / \delta P$ (see Appendix A). In what follows it might be helpful to interpret the expressions $\mathbf{X}^{\text {th }}, F$, and 
$\left\langle\mathbf{X}^{\text {th }} \cdot \mathbf{I}\right\rangle$ from the perspective of linear nonequilibrium thermodynamics [18], Sec. 4.5.1. Accordingly, $\mathbf{X}^{\text {th }}$ can be regarded as a thermodynamic force. Changes of the free energy $F$ can be decomposed like $\mathrm{d} F=\mathrm{d} U-T \mathrm{~d} S$ with $\mathrm{d} S=\mathrm{d}_{i} S+\mathrm{d}_{e} S$, where $\mathrm{d}_{i} S$ and $\mathrm{d}_{e} S$ describe entropy changes due to so-called internal and external processes. In general, internal processes are processes that would also be present if we isolate a system from its environment, whereas $\mathrm{d}_{e} S$ is given by $T \mathrm{~d}_{e} S=\mathrm{d} U$. Consequently, we have $\mathrm{d} F=-T \mathrm{~d}_{i} S$ and Eq. (10) can be written as

$$
\mathrm{d}_{i} S=\frac{1}{T}\left\langle\mathbf{X}^{\text {th }} \cdot \mathbf{I}\right\rangle \mathrm{d} t+\frac{\gamma}{T} \int P\left|\frac{\partial}{\partial \mathbf{p}} \frac{\delta F}{\delta P}\right|^{2} \mathrm{~d} p^{3} \mathrm{~d} q^{3} \mathrm{~d} t .
$$

Accordingly, the two terms occurring in Eq. (11) describe two ways of internal entropy production. However, the first term involves a conservative (reversible) force $\mathbf{I}$. Therefore, it cannot contribute to the entropy production $\mathrm{d}_{i} S$ and we conclude that $\left\langle\mathbf{X}^{\text {th }} \cdot \mathbf{I}\right\rangle=0$ for solutions $P(\mathbf{p}, \mathbf{q}, t)$ of Eq. (9) (see also [18], Sec. 4.5.5, and the null space hypothesis of the GENERIC approach to thermodynamics [22]). Putting $\left\langle\mathbf{X}^{\text {th }} \cdot \mathbf{I}\right\rangle$ equal to zero in Eq. (11), we see that entropy changes due to internal processes can result either in an entropy increase or leave the entropy constant: $\mathrm{d}_{i} S \geq 0$. In fact, the inequality $\mathrm{d}_{i} S \geq 0$ should not be regarded as a conclusion but as a fundamental thermodynamical principle. In this sense, we have just shown that the nonlinear Vlasov model (4) satisfies this principle of internal entropy increase. Let us put now $\left\langle\mathbf{X}^{\text {th }}\right.$. I $)$ equal to zero in Eq. (10). Then, we obtain

$$
\frac{\mathrm{d}}{\mathrm{d} t} F=-\gamma \int P\left|\frac{\partial}{\partial \mathbf{p}} \frac{\delta F}{\delta P}\right|^{2} \mathrm{~d} p^{3} \mathrm{~d} q^{3} \leq 0
$$

and conclude that the free energy is a monotonically decreasing function. That is, the free energy decrease may be regarded as a consequence of the internal entropy increase. Alternatively, taking a mathematical point of view, it can be shown that $\left\langle\mathbf{X}^{\text {th }} \cdot \mathbf{I}\right\rangle=0$ holds if we have $\nabla \cdot \mathbf{I}=0$ and $\mathbf{I} \cdot \nabla \delta U / \delta P=0$ [18], Sec. 4.5.5. In fact, these conditions are satisfied by the expressions occurring in Eqs. (1)-(3). Equation (12) can be regarded as a counterpart to the first issue $\mathrm{d} L / \mathrm{d} t \leq 0$ mentioned in Sec. II A 1 .

Let us turn now to the second issue mentioned in the simple example of Sec. II A 1. To this end, we first note that the implication

$$
\frac{\mathrm{d}}{\mathrm{d} t} F=0 \Leftrightarrow \frac{\partial}{\partial t} P=0
$$

holds (see Appendix B). That is, the stationarity of the free energy implies the stationarity of the probability density and vice versa. Substituting Eqs. (5) and (6) into Eq. (8), we see that stationary distributions $P_{\mathrm{st}}$ are given by extrema of the free energy $F$. That is, the variational derivative of $F$ with respect to $P$ is a constant at $P=P_{\mathrm{st}}$ :

$$
\left.\frac{\delta F}{\delta P}\right|_{P=P_{\mathrm{st}}}=\mu
$$

with $\mu=k_{B} T\left\{1-\ln \left[\left(2 \pi k_{B} T / m\right)^{3 / 2} Z\right]\right\}$. This is in analogy to the aforementioned simple case of Lyapunov's direct method for which we have seen that extrema of $L$ correspond to fixed points $x_{\mathrm{st}}$. Note that Eq. (14) can alternatively be expressed by stating that the first variation $\delta F$ vanishes at stationary distributions for all perturbations $\epsilon: \delta F\left[P_{\mathrm{st}}\right](\epsilon)=0$.

In many cases $F$ is bounded from below ([18], Sec. 4.7). In these cases, $F$ corresponds to a Lyapunov functional and we conclude that in the long time limit any transient probability density $P(\mathbf{p}, \mathbf{q}, t)$ of Eq. (4) will converge to a stationary one (H-theorem). In doing so, we see that the third issue listed in Sec. II A 1 can be generalized such that it also holds for the nonlinear Vlasov model (4).

Turning to the final point mentioned in Sec. II A 1, we note that irrespective of the boundedness of $F$, the free energy functional $F$ can be used to determine the stability of stationary distributions $P_{\mathrm{st}}(\mathbf{p}, \mathbf{q})$. If $P_{\mathrm{st}}$ corresponds to a free energy minimum then it is stable; if $P_{\text {st }}$ corresponds to a free energy maximum or a saddle point then it is unstable (see [18]. Sec. 5.1.1, and [19]):

$$
\begin{aligned}
& \delta^{2} F>0 \Rightarrow P_{\text {st }}(\mathbf{p}, \mathbf{q})=\text { stable } \\
& \delta^{2} F<0 \Rightarrow P_{\text {st }}(\mathbf{p}, \mathbf{q})=\text { unstable }
\end{aligned}
$$

Note that in Eq. (15a) we require that for all perturbations the inequality $\delta^{2} F>0$ holds, whereas in Eq. (15b) it is sufficient that there is at least one perturbation for which the inequality $\delta^{2} F<0$ holds.

\section{Stability analysis using Lyapunov's direct method in the reduced phase space}

Our next objective is to show that the nature of the extrema (i.e. the sign of the second variation $\delta^{2} F$ ) can be determined by studying an appropriately defined reduced problem that can be derived from the full dynamics given by Eq. (3). More precisely, we will show next that there is no need to carry out a stability analysis in the sixdimensional phase space $(\mathbf{p}, \mathbf{q})$. It is sufficient to consider the subspace which is spanned by the position variable $\mathbf{q}$.

First, let us decompose for $P_{\mathrm{st}}(\mathbf{p}, \mathbf{q})=\sigma_{\mathrm{st}}(\mathbf{p}) \rho_{\mathrm{st}}(\mathbf{q})$ the free energy $F$ into $F\left[P_{\mathrm{st}}(\mathbf{p}, \mathbf{q})\right]=F_{p}\left[\sigma_{\mathrm{st}}(\mathbf{p})\right]+F_{q}\left[\rho_{\mathrm{st}}(\mathbf{q})\right]$ with $\quad F_{p}=\left\langle p^{2} /(2 m)\right\rangle-T S_{p}, \quad F_{q}=U_{\text {pot }}-T S_{q}$, $\delta U_{\mathrm{pot}} / \delta \rho=V, \quad S_{p}=-k_{B} \int \sigma \ln \sigma \mathrm{d} p^{3}, \quad$ and $\quad S_{q}=$ $-k_{B} \int \rho \ln \rho \mathrm{d} q^{3}$. Then, the stationary distributions $\sigma_{\mathrm{st}}(\mathbf{p})$ and $\rho_{\text {st }}(\mathbf{q})$ given by Eqs. (5) and (6) satisfy the extremal principles $\delta F_{p} / \delta \sigma_{\mathrm{st}}(\mathbf{p})=\mu_{p}$ and $\delta F_{q} / \delta \rho_{\mathrm{st}}(\mathbf{q})=\mu_{q}$, where $\mu_{p}$ and $\mu_{q}$ are normalization constants that may be interpreted as chemical potentials. In other words, $\sigma_{\text {st }}(\mathbf{p})$ and $\rho_{\text {st }}(\mathbf{q})$ correspond to the extrema of the (subsystem) free energies $F_{p}$ and $F_{q}$. Likewise, for $P_{\text {st }}(\mathbf{p}, \mathbf{q})$ we have $\delta F / \delta P_{\mathrm{st}}(\mathbf{p}, \mathbf{q})=\mu=\mu_{p}+\mu_{q}$. Having discussed 
the first-order variational derivatives, we turn now to the second-order derivatives. The second variation of $F$ with respect to a perturbation $\epsilon$ reads

$$
\delta^{2} F\left[P_{\mathrm{st}}(\mathbf{p}, \mathbf{q})\right][\epsilon(\mathbf{p}, \mathbf{q})]=\delta^{2} U+k T \int \frac{\epsilon^{2}(\mathbf{p}, \mathbf{q})}{P_{\mathrm{st}}(\mathbf{p}, \mathbf{q})} \mathrm{d} p^{3} \mathrm{~d} q^{3}
$$

with

$$
\delta^{2} U=\int G\left(\mathbf{q}, \mathbf{q}^{\prime}\right) \tilde{\boldsymbol{\epsilon}}(\mathbf{q}) \tilde{\boldsymbol{\epsilon}}\left(\mathbf{q}^{\prime}\right) \mathrm{d} q^{3} \mathrm{~d} q^{13}
$$

and $\tilde{\boldsymbol{\epsilon}}(\mathbf{q})=\int \epsilon(\mathbf{p}, \mathbf{q}) \mathrm{d} p^{3}$. Now we use the Schwarz inequality for integrals in the form of $\int a^{2}(x) \mathrm{d} x \int b^{2}(x) \mathrm{d} x \geq$ $\left[\int a(x) b(x) \mathrm{d} x\right]^{2}$ and make the following replacements: $\mathrm{d} x \rightarrow \mathrm{d} p^{3}, a \rightarrow \epsilon(\mathbf{p}, \mathbf{q}) / \sqrt{\sigma_{\text {st }}(\mathbf{p})}, b \rightarrow \sqrt{\sigma_{\text {st }}(\mathbf{p})}$. Thus, we get

$$
\int \frac{\epsilon^{2}(\mathbf{p}, \mathbf{q})}{\sigma_{\mathrm{st}}(\mathbf{p})} \mathrm{d} p^{3} \geq\left[\int \epsilon(\mathbf{p}, \mathbf{q}) \mathrm{d} p^{3}\right]^{2}=\tilde{\epsilon}^{2}(\mathbf{q}) .
$$

Substituting this result into Eq. (16), we obtain

$$
\begin{aligned}
\delta^{2} F\left[P_{\mathrm{st}}(\mathbf{p}, \mathbf{q})\right][\epsilon(\mathbf{p}, \mathbf{q})] & \geq \delta^{2} F_{q}\left[\rho_{\mathrm{st}}(\mathbf{q})\right][\tilde{\boldsymbol{\epsilon}}(\mathbf{q})] \\
& =\delta^{2} U+k T \int \frac{\tilde{\boldsymbol{\epsilon}}^{2}(\mathbf{q})}{\rho_{\mathrm{st}}(\mathbf{q})} \mathrm{d} q^{3} .
\end{aligned}
$$

The inequality (19) represents our central result. From Eq. (19) it follows that, if $\delta^{2} F_{q}>0$ holds for all perturbations $\tilde{\boldsymbol{\epsilon}}(\mathbf{q})$, then $\delta^{2} F>0$ holds for all perturbations $\epsilon(\mathbf{p}, \mathbf{q})$ and $P_{\mathrm{st}}(\mathbf{p}, \mathbf{q})$ is a stable stationary distribution. The equals sign in Eqs. (18) and (19) holds for perturbations of the form $\epsilon(\mathbf{p}, \mathbf{q})=\sqrt{\sigma_{\mathrm{st}}(\mathbf{p})} \tilde{\boldsymbol{\epsilon}}(\mathbf{q})$. Consequently, if there is a perturbation $\tilde{\boldsymbol{\epsilon}}(\mathbf{q})$ such that $\delta^{2} F_{q}<0$ holds, then for the perturbation $\boldsymbol{\epsilon}(\mathbf{p}, \mathbf{q})=\sqrt{\sigma_{\text {st }}(\mathbf{p})} \tilde{\boldsymbol{\epsilon}}(\mathbf{q})$ we have $\delta^{2} F<0$ and $P_{\text {st }}(\mathbf{p}, \mathbf{q})$ is an unstable stationary distribution. In sum, the inequality (19) states that the relations (15) can be simplified and replaced by

$$
\begin{aligned}
& \delta^{2} F_{q}>0 \Rightarrow P_{\text {st }}(\mathbf{p}, \mathbf{q})=\text { stable } \\
& \delta^{2} F_{q}<0 \Rightarrow P_{\text {st }}(\mathbf{p}, \mathbf{q})=\text { unstable. }
\end{aligned}
$$

We see that the free energy functional $F_{q}$ defined on the subspace $\mathbf{q}$ determines completely the stability of the stationary distributions of the full dynamical system.

\section{Stability analysis using Vlasov-Smoluchowski equations}

Equation (20) states that the stability analysis of $P_{\mathrm{st}}(\mathbf{p}, \mathbf{q})$ can be based on the reduced free energy measure $F_{q}$. Therefore, the question arises: how can we interpret this approach via $F_{q}$ ? What is the dynamical system that is represented by $F_{q}$ ? We will answer this question by introducing heuristically an evolution equation related to $F_{q}$. To this end, we first introduce a new time variable $\tau$. Let $\tilde{\rho}(\mathbf{q}, \tau)$ denote the probability density of a dynamical sys- tem exhibiting the free energy $F_{q}$. According to the free energy approach to nonlinear Fokker-Planck equations [18], Sec. 4.5 , the evolution of $\tilde{\rho}(\mathbf{q}, \tau)$ is given by

$$
\frac{\mathrm{d}}{\mathrm{d} \tau} \tilde{\rho}(\mathbf{q}, \tau)=-\frac{\partial}{\partial \mathbf{q}}\left(\tilde{\rho}(\mathbf{q}, \tau) \frac{\partial}{\partial \mathbf{q}} \frac{\delta F_{q}}{\delta \tilde{\rho}(\mathbf{q}, \tau)}\right) .
$$

It can be shown that - consistent with fundamental principles in physics - transient solutions $\tilde{\rho}(\mathbf{q}, \tau)$ of Eq. (21) converge to stationary distributions $\tilde{\rho}_{\text {st }}(\mathbf{q})$ that make $F_{q}$ minimal [18], Sec. 4.5.7. Therefore, we conclude that stationary distributions $\tilde{\rho}_{\mathrm{st}}(\mathbf{q})$ of Eq. (21) corresponds to stationary distributions $\rho_{\mathrm{st}}(\mathbf{q})$ of the original Vlasov model (3). Moreover, we conclude that stationary distributions $\tilde{\rho}_{\text {st }}(\mathbf{q})$ are stable (unstable) if the corresponding distributions $P_{\text {st }}(\mathbf{p}, \mathbf{q})=\sigma_{\mathrm{st}}(\mathbf{p}) \tilde{\rho}_{\mathrm{st}}(\mathbf{q})$ are stable (unstable). In other words, the full and reduced Vlasov Fokker-Planck equations (3) and (21) exhibit stationary solutions that are not only equivalent to each other but also have the same stability properties. As a result, if we know the stability of $\tilde{\rho}_{\text {st }}(\mathbf{q})$, we can conclude whether or not $F_{q}$ exhibits a minima at $\tilde{\rho}_{\mathrm{st}}(\mathbf{q})$ and, in doing so, we can determine the stability of $P_{\text {st }}(\mathbf{p}, \mathbf{q})$, see Eq. (20). In sum, stability analysis can be performed on the basis of the reduced Vlasov equation (21) using the implications

$\tilde{\rho}_{\mathrm{st}}(\mathbf{q})=$ stable $\Rightarrow P_{\mathrm{st}}(\mathbf{p}, \mathbf{q})=\sigma_{\mathrm{st}}(\mathbf{p}) \tilde{\rho}_{\mathrm{st}}(\mathbf{q})=$ stable

$\tilde{\rho}_{\mathrm{st}}(\mathbf{q})=$ unstable $\Rightarrow P_{\mathrm{st}}(\mathbf{p}, \mathbf{q})=\sigma_{\mathrm{st}}(\mathbf{p}) \tilde{\rho}_{\mathrm{st}}(\mathbf{q})=$ unstable

instead of the relations (20).

Having derived the evolution equation (21), we still need to discuss it physical interpretation. To this end, it is helpful to consider the self-consistent Langevin equation that corresponds to Eq. (21) and reads ([18], Sec. 3.4.2)

$$
\frac{\mathrm{d}}{\mathrm{d} \tau} \mathbf{q}(\tau)=-\frac{\partial}{\partial \mathbf{q}} V[\mathbf{q}(\tau), \tilde{\rho}(\mathbf{q}, \tau)]+\sqrt{k_{B} T} \boldsymbol{\Gamma}(\tau),
$$

where $\boldsymbol{\Gamma}$ denotes a Langevin force with $\left\langle\Gamma_{i}(\tau) \Gamma_{k}\left(\tau^{\prime}\right)\right\rangle=$ $2 \delta_{i k} \delta\left(\tau-\tau^{\prime}\right)$. The Langevin equation (23) can be derived from the original Langevin equation (3) by putting $\mathrm{d} \mathbf{p} / \mathrm{d} t=0$ and $t=\gamma \tau$. Because of this property, Eqs. (21) and (23) can be regarded as Smoluchowski equations [17] that can be obtained in the high friction limit $\gamma \rightarrow \infty$ from the second-order dynamical equation (3) and (4). A more formal treatment of this high friction limit is given in Appendix C.

As we have just stated, Smoluchowski equations are usually defined for the high friction limit $\gamma \rightarrow \infty$. In contrast, the derivation of Eq. (21) holds for arbitrary values of $\gamma>0$. That is, $\gamma$ can be small and even represent a perturbation parameter. In the analysis carried out above, the Smoluchowski equation (21) is just the nonlinear Fokker-Planck equation related to the free energy functional $F_{q}$. The stability analysis by means of the Smoluchowski equation (21) and the relation (22) can be 
carried out whether $\gamma$ is large or not. It should be regarded as a technique alternative to the free energy approach that has been discussed in Sec. II A 3 and was centered around the relation (20).

\section{B. Haïssinski theory}

\section{Vlasov-Fokker-Planck equation of the Haïssinski model}

Following the notation used in [5,9], we describe bunched particles of a longitudinal beam in a storage ring by means the relative particle position $q$ with respect to the bunch center and the relative particle energy $p$ (or energy error) with respect to the beam design energy. Note that we are dealing now with a two-dimensional phase space $(p, q)$ instead of a six-dimensional one. Accordingly, the probability density of $p$ and $q$ can be computed from $P(p, q, t)=\langle\delta[q-q(t)] \delta[p-p(t)]\rangle$ and the corresponding reduced probability densities are given by $\sigma(p, t)=\langle\delta[p-p(t)]\rangle$ and $\rho(q, t)=\langle\delta[q-q(t)]\rangle$. The single particle potential $V_{0}$ reads $V_{0}(q)=q^{2} / 2$ such that the Hamiltonian (1) becomes $H=p^{2} / 2+q^{2} / 2+$ $\int G\left(q, q^{\prime}\right) \rho\left(q^{\prime}, t\right) \mathrm{d} q^{\prime}$, where we have put $m=1$. The integral kernel $G(z)$ corresponds to the wake field function $W(z)$ of storage rings like $G(z)=\int^{z} W\left(z^{\prime}\right) \mathrm{d} z^{\prime}$. Aiming at a discussion of Haïssinski distributions $P(p, q)$, we consider a wake field function $W(z)=\kappa \mathrm{d} \delta(z) / \mathrm{d} z$, which is said to be dominated by a purely imaginary impedance ([7], Sec. 6.2), where $\kappa$ can assume both positive and negative values [7,9,23]. Consequently, we have $G\left(q, q^{\prime}\right)=\kappa \delta(q-$ $\left.q^{\prime}\right)$ and the self-consistent potential $V$ reads $V[q, \rho]=$ $q^{2} / 2+\kappa \rho(q, t)$. In sum, the nonlinear Vlasov-FokkerPlanck Eq. (4) becomes [18,24-26]

$$
\begin{aligned}
\frac{\partial}{\partial t} P(p, q, t)= & p \frac{\partial}{\partial q} P(p, q, t) \\
& -\left[q+\kappa \frac{\partial \rho(q, t)}{\partial q}\right] \frac{\partial}{\partial p} P(p, q, t) \\
& +\underbrace{\gamma \frac{\partial}{\partial p}\left(p+Q \frac{\partial}{\partial p}\right) P(p, q, t)}_{A},
\end{aligned}
$$

where $Q$ is a nonequilibrium noise amplitude and replaces the equilibrium expression $k_{B} T$. The Fokker-Planck collision operator $A$ in Eq. (24) accounts for dissipation and fluctuations due to synchrotron radiation $[4,5,8,23,27,28]$.

\section{Stability analysis using Lyapunov's direct method in the reduced phase space}

Let us apply the results derived in Sec. II A in order to examine stationary solutions of the beam dynamics model (24). First, we note that as shown in [24] the stationary distribution of Eq. (24) can be expressed as $P_{\mathrm{st}}(p, q)=$ $\sigma_{\text {st }}(p) \rho_{\text {st }}(q)$ with $\sigma_{\text {st }}(p)=\sqrt{2 \pi Q} \exp \left\{-p^{2} /(2 Q)\right\}-1$, where $\rho_{\text {st }}(q)$ denotes the Haïssinski distribution [29] de- fined by

$$
\left[Q+\kappa \rho_{\mathrm{st}}(q)\right] \frac{\mathrm{d} \rho_{\mathrm{st}}(q)}{\mathrm{d} q}=-q \rho_{\mathrm{st}}(q)
$$

As argued in Sec. II A 2, the stability of $P_{\mathrm{st}}(p, q)$ is determined by the free energy

$$
F[P(p, q)]=\left\langle\frac{p^{2}+q^{2}}{2}\right\rangle+\frac{\kappa}{2} \int \rho^{2}(q) \mathrm{d} q-Q S
$$

with $S[P(p, q)]=-\int P \ln P \mathrm{~d} p \mathrm{~d} q$. However, as shown in Sec. II A 3, it is sufficient to analyze only the subspace free energy $F_{q}$ defined by

$$
F_{q}[\rho(q)]=\left\langle\frac{q^{2}}{2}\right\rangle+\frac{\kappa}{2} \int \rho^{2}(q) \mathrm{d} q-Q S_{q}
$$

with $S_{q}[\rho(q)]=-\int \rho \ln \rho \mathrm{d} q$. In particular, we need to determine whether or not the Haïssinski distribution $\rho_{\mathrm{st}}(q)$ corresponds to a minimum of the free energy $F_{q}$. To this end, we examine the second variation of $F_{q}$ with respect to a perturbation $\tilde{\epsilon}$. From Eq. (27) we find that $\delta^{2} F_{q}=\int\left[\kappa+Q / \rho_{\mathrm{st}}(q)\right] \tilde{\epsilon}^{2}(q) \mathrm{d} q$. Using Shiino's expansion $[18,19] \tilde{\epsilon}(q)=\sqrt{\rho_{\mathrm{st}}(q)} \chi(q)$ that involves a new perturbation function $\chi$, we obtain

$$
\delta^{2} F_{q}(\chi)=\int\left[\kappa \rho_{\mathrm{st}}(q)+Q\right] \chi^{2}(q) \mathrm{d} q
$$

For $\kappa>0$ we have $\delta^{2} F_{q}>0$. That is, the Haïssinski distribution corresponds to a stable stationary probability density. Let us consider the case $\kappa<0$. If there is a $q^{*}$-value for which $\kappa \rho_{\text {st }}\left(q^{*}\right)+Q<0$ holds, then there is a formally defined perturbation $\chi$ with $\chi^{2}(q)=\delta\left(q-q^{*}\right)$ for which $\delta^{2} F$ becomes negative. Therefore, the Haïssinski distribution is an unstable distribution. Following $[9,23]$, we consider the case $\kappa<0$ but $\kappa \rho_{\text {st }}(q)+Q>0$ for all $q$. Then, from Eq. (25) if follows that $\rho_{\mathrm{st}}(q)$ is unimodal and symmetric with a peak at $q=0$. Consequently, if $\kappa \rho_{\text {st }}(q)+Q>0$ holds for the maximum value $q=0$, then $\kappa \rho_{\text {st }}(q)+Q>0$ holds for all $q$. Conversely, if $\kappa \rho_{\text {st }}(0)+Q<0$ holds, then there are several values $q^{*}$ (first of all $q^{*}=0$ ) for which $\kappa \rho_{\text {st }}(q)+Q<0$ and the distribution (if it exists) is an unstable one. In sum, in analogy to Eq. (20), our stability analysis for the VlasovFokker-Planck equation (24) yields

$$
\begin{aligned}
& \kappa \rho_{\mathrm{st}}(q=0)+Q>0 \Rightarrow \delta^{2} F_{q}>0 \Rightarrow P_{\mathrm{st}}(p, q)=\text { stable } \\
& \kappa \rho_{\mathrm{st}}(q=0)+Q<0 \Rightarrow \delta^{2} F_{q}<0 \Rightarrow P_{\mathrm{st}}(p, q)=\text { unstable. }
\end{aligned}
$$

This result is consistent with previous studies on the stability of Haïssinski distributions [8,9]. For continuous 
Haïssinski distributions we have $\kappa \rho_{\mathrm{st}}(q)+Q>0$ for all $q .{ }^{1}$ As a result, continuous Haïssinski distributions are stable if they exist. In fact, considering $\kappa$ as a control parameter and decreasing $\kappa$, then continuous Haïssinski distributions cease to exist at the instability point given by $\kappa \rho_{\text {st }}(q=0)+Q=0$ (see also [9,23,27]). Continuous distributions $\rho_{\text {st }}(q)$ can be computed from [24,30,31]

$$
\rho_{\mathrm{st}}(q)=\frac{Q}{\kappa} \operatorname{LW}\left(\frac{\kappa}{Z Q} \Phi(q)\right)
$$

where $\operatorname{LW}(\cdot)$ is a distortion function given by the Lambert's W-function [32], $\Phi$ is the Gaussian distribution $\Phi(q)=[\sqrt{2 \pi Q}]^{-1} \exp \left\{-q^{2} /(2 Q)\right\}$, and $Z$ is determined by the requirement $\int \rho_{\mathrm{st}}(q) \mathrm{d} q=1$. Using this function, we can compute $\rho_{\text {st }}(0)$ and the critical parameter value $\kappa_{c}$ defined by $\kappa_{c} \rho_{\mathrm{st}, \kappa_{c}}(0)+Q=0$ or

$$
\kappa_{c}=-\frac{Q}{\rho_{\mathrm{st}, \kappa_{c}}(0)} .
$$

An analytical expression for $\kappa_{c}$ can be found and is given by [23]

$$
\kappa_{c}=-\sqrt{2} Q^{3 / 2} \int_{0}^{1} \frac{1-x}{\sqrt{x-\ln x-1}} \mathrm{~d} x
$$

A geometrical interpretation of Eq. (31) can be obtained as follows. It is known that $\rho_{\text {st }}(q)$ describes bunch shortening for $\kappa<0$. That is, $\rho_{\mathrm{st}}(0)$ increases as a function of $|\kappa|$ for $\kappa<0$ - as illustrated in Fig. 1 -and $Q / \rho_{\text {st }}(0)$ decreases - as shown in Fig. 2. Consequently, if we plot $f_{1}(\kappa)=Q / \rho_{\mathrm{st}, \kappa}(0)$ as a function of $\kappa$, then the critical parameter $\kappa_{c}$ is defined by the intersection point of $f_{1}(\kappa)$ with the diagonal $f_{2}(\kappa)=\kappa$ (see Fig. 2).

\section{Stability analysis using the Smoluchowski equation of the Haïsinski model}

Let us reiterate that the stability analysis carried out in the previous section IIB 2 can be interpreted as a Smoluchowski approach. To put emphasis on this point, we would like to note that the Smoluchowski equation related to the free energy (27) explicitly reads

$$
\begin{aligned}
\frac{\mathrm{d}}{\mathrm{d} \tau} \tilde{\rho}(q, \tau)= & \frac{\partial}{\partial q}\left[q+\kappa \frac{\partial \tilde{\rho}(q, \tau)}{\partial q}\right] \tilde{\rho}(q, \tau) \\
& +Q \frac{\partial^{2}}{\partial q^{2}} \tilde{\rho}(q, \tau) .
\end{aligned}
$$

\footnotetext{
${ }^{1}$ For continuous distributions it follows from Eq. (25) that the relation $\kappa \rho_{\text {st }}(q)+Q \neq 0$ must be satisfied, which implies that we either have $\kappa \rho_{\text {st }}(q)+Q>0$ or $\kappa \rho_{\text {st }}(q)+Q<0$ for all $q$. The latter option does not allow for continuous distributions that can be normalized to unity.
}

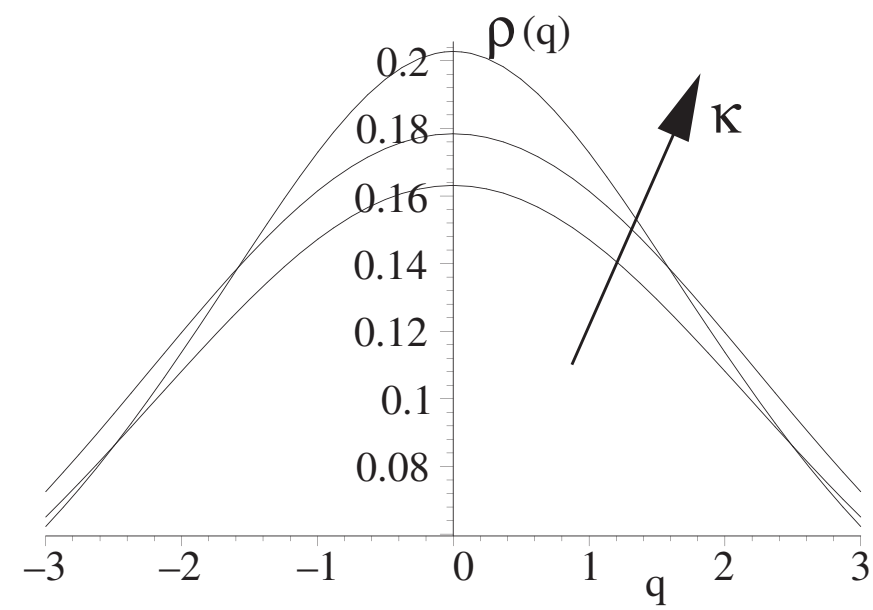

FIG. 1. Bunch shortening due to a decrease of the parameter $\kappa$. Haïssinski distributions (30) are shown for several parameters $\kappa$ (from bottom to top: $\kappa_{1}=0, \kappa_{2}=-5.0, \kappa_{3}=-10.0$ ) and $Q=$ 5.0 .

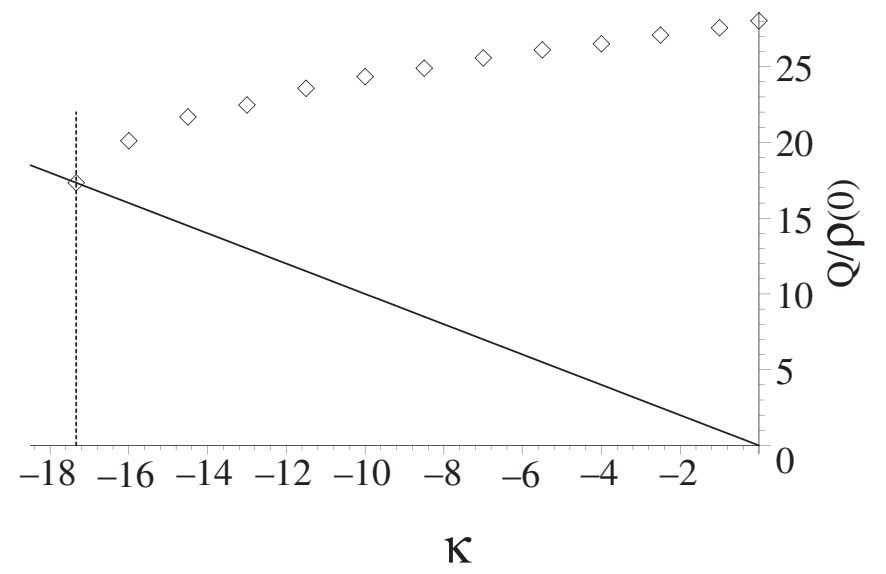

FIG. 2. Geometrical solution of Eq. (31) for $Q=5.0$. Diamonds: $f_{1}(\kappa)=Q / \rho_{\text {st }}(0)$ computed from Eq. (30). Solid line: diagonal $f_{2}(\kappa)=\kappa$. Dashed vertical line: analytical solution $\kappa_{c}$ computed from Eq. (32).

Every stationary distribution $\tilde{\rho}_{\text {st }}$ of Eq. (33) corresponds to a Haïssinski distribution (25): $\tilde{\rho}_{\mathrm{st}}=\rho_{\mathrm{st}}$. Moreover, the distributions $\tilde{\rho}_{\text {st }}$ and $\rho_{\text {st }}$ have the same stability properties. Consequently, the stability analysis in Sec. II B 2 that was centered around the free energy $F_{q}$ [see Eq. (27)] and has led us to Eq. (29) actually was a stability analysis for stationary solutions of the Smoluchowski model (33). However, in principle, any other type of stability analysis can be applied to the Smoluchowski model (33). For example, we may apply linear stability analysis.

In order to make this point more explicit, let us briefly outline the linear stability analysis of stationary solutions of Eq. (33). Let $\tilde{\boldsymbol{\epsilon}}(q, \tau)$ denote a small perturbation of the stationary distribution $\tilde{\rho}_{\mathrm{st}}(q)=\rho_{\mathrm{st}}(q)$ given by Eq. (25). That is, we put $\tilde{\rho}(q, \tau)=\rho_{\mathrm{st}}(q)+\tilde{\boldsymbol{\epsilon}}(q, \tau)$. From Eq. (33) it then follows that 


$$
\frac{\mathrm{d}}{\mathrm{d} \tau} \tilde{\boldsymbol{\epsilon}}(q, \tau)=\frac{\partial}{\partial q} q \tilde{\boldsymbol{\epsilon}}(q, \tau)+\frac{\partial^{2}}{\partial q^{2}}\left\{\left[\kappa \rho_{\mathrm{st}}(q)+Q\right] \tilde{\epsilon}(q, \tau)\right\}
$$

neglecting terms of second and higher order in $\tilde{\boldsymbol{\epsilon}}$. Using Eq. (25) in terms of $q=-\left[\kappa+Q / \rho_{\text {st }}\right] \mathrm{d} \rho_{\text {st }} / \mathrm{d} q$, we obtain

$$
\begin{aligned}
\frac{\mathrm{d}}{\mathrm{d} \tau} \tilde{\boldsymbol{\epsilon}}(q, \tau)= & -\frac{\partial}{\partial q}\left\{\left[\kappa+\frac{Q}{\rho_{\mathrm{st}}(q)}\right] \frac{\mathrm{d} \rho_{\mathrm{st}}}{\mathrm{d} q} \tilde{\boldsymbol{\epsilon}}(q, \tau)\right\} \\
& +\frac{\partial^{2}}{\partial q^{2}}\left\{\left[\kappa+\frac{Q}{\rho_{\mathrm{st}}(q)}\right] \rho_{\mathrm{st}}(q) \tilde{\boldsymbol{\epsilon}}(q, \tau)\right\},
\end{aligned}
$$

which can be transformed into

$$
\frac{\mathrm{d}}{\mathrm{d} \tau} \tilde{\boldsymbol{\epsilon}}(q, \tau)=\frac{\partial}{\partial q} \rho_{\mathrm{st}}(q) \frac{\partial}{\partial q}\left\{\left[\kappa+\frac{Q}{\rho_{\mathrm{st}}(q)}\right] \tilde{\boldsymbol{\epsilon}}(q, \tau)\right\} .
$$

If $\kappa+Q / \rho_{\mathrm{st}}(q)>0$ holds for all $q$, that is, if $\kappa>\kappa_{c}$ (cf. Sec. II B 2), then Eq. (36) corresponds to a diffusion equation with a positive-definite (state-dependent) diffusion term and we conclude that for $t \rightarrow \infty$ the perturbation $\tilde{\boldsymbol{\epsilon}}$ will converge to zero everywhere. In this case, we conclude that $\rho_{\text {st }}(q)$ is a stable stationary distribution. In contrast, if $\kappa+Q / \rho_{\mathrm{st}}(q)<0$ holds for $q \in I$, where $I$ denotes a particular interval [i.e. if there is a noncontinuous distribution $\rho_{\mathrm{st}}(q)$ with $\kappa<\kappa_{c}$, then the coefficient $\kappa+$ $Q / \rho_{\mathrm{st}}(q)$ is negative on $I$. Consequently, the perturbation will increase on $I$. In this case, we conclude that $\rho_{\text {st }}(q)$ is an unstable stationary distribution. Note that this argument holds irrespective of the fact that the perturbation $\tilde{\epsilon}$ exhibits both negative and positive function values. An alternative proof of this line of reasoning can be found in Appendix D. Using Eq. (22), we conclude from the linear stability analysis of the Smoluchowski model (33) that $P_{\mathrm{st}}(\mathbf{p}, \mathbf{q})=\sigma_{\mathrm{st}}(\mathbf{p}) \rho_{\mathrm{st}}(\mathbf{q})$ is a stable (unstable) solution for $\kappa>\kappa_{c}\left(\kappa<\kappa_{c}\right)$. That is, by means of a linear stability analysis of the Smoluchowski model we arrived at the same conclusions as in Sec. II B 2.

\section{CONCLUSIONS}

We have shown that the stability of stationary distributions of particular six-dimensional nonlinear VlasovFokker-Planck equations can be determined by studying the stability of stationary distributions of appropriately defined Smoluchowski equations defined on reduced three-dimensional phase spaces. The stability analysis for the Smoluchowski equations can in principle be carried out by different methods: linear stability analysis, analysis of self-consistency equations for order parameters (see e.g. [18], Sec. 5.1.3), and Lyapunov's direct method. That is, irrespective of the method that is used to determine the stability of the reduced Smoluchowski dynamics, the conclusion can be drawn that the full dynamics evolving in the six-dimensional phase space has the same stability properties as the reduced dynamics. In particular, in the context of nonlinear Vlasov-Fokker-Planck equations often linear stability analysis is used. Irrespective of the fact that in our analysis Lyapunov's direct method has been used to construct a link between full nonlinear models and reduced Smoluchowski models, linear stability analysis can definitely be applied to determine the stability of stationary distributions. The advantage of our approach is that such a linear stability analysis will involve only simplified, reduced partial differential operators. This point has been demonstrated explicitly in Sec. II B 3.

It is important to realize, however, that the unstable, nonstationary behavior of the reduced dynamics will in general not correspond to the unstable, nonstationary behavior of the full dynamics. Likewise, amplitude equations that describe the growth of unstable modes and are derived from the full and the reduced dynamics will in general not be equivalent.

We studied bunch particle distributions of the Haiissinski type. We showed that they correspond to extrema of free energy functionals. Continuous distributions have been found to be stable and to correspond to free energy minima. We demonstrated that, at the critical parameter $\kappa_{c}$ at which continuous solutions cease to exist, the free energy minima become maxima or saddle points. That is, discontinuous stationary distributions that may exist for $\kappa<\kappa_{c}$ will correspond to unstable distributions. These results are in line with previous analytical and numerical studies that have been shown that for $\kappa>\kappa_{c}$ stable Haïssinski distributions exist, whereas for $\kappa<\kappa_{c}$ beam dynamics defined by the Vlasov-Fokker-Planck equations of the Haïssinski type becomes unstable $[8,9]$.

We would like to note that for the class of nonlinear Vlasov-Fokker-Planck equations addressed in our study nonlinear Smoluchowski equations can be derived simply by putting $\mathrm{d} \mathbf{p} / \mathrm{d} t=0$ or by more sophisticated methods (see Appendix C). For more comprehensive beam dynamic models it is however not at all clear whether associated Smoluchowski models can be derived. This is because it is still an open question how to derive in general Smoluchowski equations from nonlinear Fokker-Planck equations. In this context, we would also like to mention that our analysis was developed for many-particle systems and beam dynamic instabilities involving symmetric selfinteraction potentials and odd wake field functions, respectively. Future work may be devoted to clarify to which extent our approach can be generalized to account for asymmetric self-interaction potentials and wake field functions exhibiting even components.

\section{APPENDIX A: DERIVATION OF EQ. (10)}

In general, differentiating a free energy functional $F[P(\mathbf{p}, \mathbf{q}, t)]$ with respect to time $t$ yields

$$
\frac{\mathrm{d}}{\mathrm{d} t} F=\int \frac{\delta F}{\delta P} \frac{\partial P}{\partial t} \mathrm{~d} p^{3} \mathrm{~d} q^{3} .
$$

Substituting now the special case of Eq. (9) into Eq. (A1), 
we obtain

$$
\begin{aligned}
\frac{\mathrm{d}}{\mathrm{d} t} F= & -\int \frac{\delta F}{\delta P} \nabla \cdot(\mathbf{I} P) \mathrm{d} p^{3} \mathrm{~d} q^{3} \\
& +\gamma \int \frac{\delta F}{\delta P} \frac{\partial}{\partial \mathbf{p}}\left(P \frac{\partial}{\partial \mathbf{p}} \frac{\delta F}{\delta P}\right) \mathrm{d} p^{3} \mathrm{~d} q^{3} .
\end{aligned}
$$

Integration by parts gives us

$$
\begin{aligned}
\frac{\mathrm{d}}{\mathrm{d} t} F= & \int P\left(\nabla \frac{\delta F}{\delta P}\right) \cdot \mathbf{I} \mathrm{d} p^{3} \mathrm{~d} q^{3} \\
& -\gamma \int P\left(\frac{\partial}{\partial \mathbf{p}} \frac{\delta F}{\delta P}\right)\left(\frac{\partial}{\partial \mathbf{p}} \frac{\delta F}{\delta P}\right) \mathrm{d} p^{3} \mathrm{~d} q^{3} .
\end{aligned}
$$

Using $\mathbf{X}^{\text {th }}=-\nabla \delta F / \delta P$, Eq. (A3) is equivalent to Eq. (10).

\section{APPENDIX B: DERIVATION OF EQ. (13)}

Since $F$ is a functional of $P$, we have $\partial P / \partial t=0 \Rightarrow$ $\mathrm{d} F / \mathrm{d} t=0$. Next, let us show that the opposite implication holds as well. It is clear from Eq. (12) that if $F$ is stationary (i.e. if $\mathrm{d} F / \mathrm{d} t=0$ holds) then we have $\partial \delta F / \delta P \partial \mathbf{p}=0$. Next, we consider a probability density $P(\mathbf{p}, \mathbf{q}, t)$ that might depend explicitly on time $t$ but leaves $F[P]$ constant in the sense that we have $\partial \delta F / \delta P \partial \mathbf{p}=0$. Substituting $\partial \delta F / \delta P \partial \mathbf{p}=0$ into Eq. (9) we see that such a probability density $P(\mathbf{p}, \mathbf{q}, t)$ must satisfy the Liouville equation

$$
\frac{\partial}{\partial t} P(\mathbf{p}, \mathbf{q}, t)=-\nabla \cdot(\mathbf{I} P) .
$$

From Eq. (B1) it follows that the probability density $P(\mathbf{p}, \mathbf{q}, t)$ either corresponds to a stationary one or describes a deterministic system. In other words, Eq. (B1) exhibits two kinds of solutions: (i) $\partial P / \partial t=0$ and (ii) $\partial P / \partial t \neq 0 \quad$ with $\quad P(\mathbf{p}, \mathbf{q}, t)=\langle\delta[\mathbf{q}-\mathbf{q}(t)] \delta[\mathbf{p}-\mathbf{p}(t)]\rangle$ and $\mathrm{d} \mathbf{q} / \mathrm{d} t=\partial H / \partial \mathbf{p}, \mathrm{d} \mathbf{p} / \mathrm{d} t=-\partial H / \partial \mathbf{q}$. Deterministic solutions of type (ii) are in contradiction with the selfconsistent Langevin equation (3). That is, the system cannot be a deterministic one for $\mathrm{d} F / \mathrm{d} t=0$. Therefore, we are left with solutions of type (i) and we conclude that the probability density $P$ corresponds to a stationary one whenever the condition $\mathrm{d} F / \mathrm{d} t=0$ holds: $\mathrm{d} F / \mathrm{d} t=0 \Rightarrow$ $\partial P / \partial t=0$. Note that the same conclusion can also be drawn using an alternative argument as proposed in [17], Sec. 6.1 (last paragraph).

\section{APPENDIX C: DERIVATION OF EQ. (21) IN THE HIGH FRICTION LIMIT}

In this Appendix, we consider the high friction limit $\gamma \rightarrow \infty$. We will show that in this limiting case the Smoluchowski equation (21) can be derived from the nonlinear Vlasov-Fokker-Planck equation (4) using the techniques developed for ordinary linear Fokker-Planck equations [33].

In a first step, we eliminate the operator $\partial / \partial \mathbf{p}$ in Eq. (4) by means of the differential operator $\partial / \partial \mathbf{q}$. To this end, we integrate left and right-hand sides of Eq. (4) over a straight line $C$ defined by $\mathbf{p}(s)=\gamma\left(\mathbf{q}(s)-\mathbf{q}_{\mathbf{0}}\right)$ in the sixdimensional phase space $(\mathbf{p}, \mathbf{q})$. Then all integrals of the form $\int_{C}(\partial / \partial \mathbf{p}-\gamma \partial / \partial \mathbf{q}) A(\mathbf{p}, \mathbf{q}) \mathrm{d} s$ will vanish. Therefore, we substitute in Eq. (4) $\partial / \partial \mathbf{p}$ like

$$
\frac{\partial}{\partial \mathbf{p}}=\left(\frac{\partial}{\partial \mathbf{p}}-\frac{1}{\gamma} \frac{\partial}{\partial \mathbf{q}}\right)+\frac{1}{\gamma} \frac{\partial}{\partial \mathbf{q}} .
$$

Thus, we obtain

$$
\begin{aligned}
\frac{\partial}{\partial t} P(\mathbf{p}, \mathbf{q}, t)= & \left(\frac{\partial}{\partial \mathbf{p}}-\frac{1}{\gamma} \frac{\partial}{\partial \mathbf{q}}\right)\left\{\frac{\gamma}{m} \mathbf{p}+\frac{\partial V}{\partial \mathbf{q}}+2 \gamma k_{B} T \frac{\partial}{\partial \mathbf{q}}\right\} P \\
& +\gamma k_{B} T\left|\frac{\partial}{\partial \mathbf{p}}-\frac{1}{\gamma} \frac{\partial}{\partial \mathbf{q}}\right|^{2} P \\
& +\frac{1}{\gamma} \frac{\partial}{\partial \mathbf{q}}\left[\frac{\partial V}{\partial \mathbf{q}}+k_{B} T \frac{\partial}{\partial \mathbf{q}}\right] P .
\end{aligned}
$$

Integrating along $C$ and putting $t=\gamma \tau$ with $P(\mathbf{p}, \mathbf{q}, t) \rightarrow$ $P(\mathbf{p}, \mathbf{q}, \tau)$ yields

$$
\frac{\mathrm{d}}{\mathrm{d} \tau} \int_{C} P(\mathbf{p}, \mathbf{q}, \tau) \mathrm{d} s=\int_{C} \frac{\partial}{\partial \mathbf{q}}\left[\frac{\partial V}{\partial \mathbf{q}}+k_{B} T \frac{\partial}{\partial \mathbf{q}}\right] P \mathrm{~d} s .
$$

Next, we assume that the kinetic dynamics is in equilibrium and consider distributions of the form $P(\mathbf{p}, \mathbf{q}, \tau)=$ $\sigma_{\text {st }}(\mathbf{p}) \rho(\mathbf{q}, \tau)$ with $\sigma_{\text {st }}(\mathbf{p})$ defined by Eq. (5). Multiplying left- and right-hand sides of Eq. (C3) with $\gamma^{2}$ and substituting $\mathbf{p}=\gamma\left(\mathbf{q}-\mathbf{q}_{\mathbf{0}}\right)$ into $\sigma_{\text {st }}(\mathbf{p})$, we get expressions like $\gamma^{2} \sigma_{\mathrm{st}}(\mathbf{p})=\gamma^{2}\left(2 \pi k_{B} T / m\right)^{3 / 2} \times$

$\exp \left\{-\gamma^{2}\left(\mathbf{q}-\mathbf{q}_{\mathbf{0}}\right)^{2} /\left(2 m k_{B} T\right)\right\}$. In the high friction limit $\gamma^{2} \rightarrow \infty$ these expressions converge to the Dirac delta function $\delta\left(\mathbf{q}-\mathbf{q}_{\mathbf{0}}\right)$ such that Eq. (C3) becomes

$$
\begin{aligned}
\frac{\mathrm{d}}{\mathrm{d} \tau} \int \delta\left(\mathbf{q}-\mathbf{q}_{0}\right) \rho(\mathbf{q}, \tau) \mathrm{d} \mathbf{q}= & \int \delta\left(\mathbf{q}-\mathbf{q}_{0}\right) \frac{\partial}{\partial \mathbf{q}}\left[\frac{\partial V}{\partial \mathbf{q}}\right. \\
& \left.+k_{B} T \frac{\partial}{\partial \mathbf{q}}\right] \rho(\mathbf{q}, \tau) \mathrm{d} \mathbf{q},
\end{aligned}
$$

where we have replaced $\mathrm{d} s$ by dq. Consequently, we have

$$
\frac{\mathrm{d}}{\mathrm{d} \tau} \rho\left(\mathbf{q}_{0}, \tau\right)=\frac{\partial}{\partial \mathbf{q}_{0}}\left[\frac{\partial V}{\partial \mathbf{q}_{0}}+k_{B} T \frac{\partial}{\partial \mathbf{q}_{0}}\right] \rho\left(\mathbf{q}_{0}, \tau\right) .
$$

This result holds for all coordinates $\mathbf{q}_{0}$, which implies that we can drop the index 0. Consequently, Eq. (C5) which is equivalent to Eq. (21) is the Smoluchowski equation of Eq. (4) in the sense that it can be obtained from Eq. (4) in the high friction limit $\gamma \rightarrow \infty$. Note that the derivation presented here cannot be carried out for nonlinear Fokker-Planck equations in general. For example, as we have seen above, the procedure requires that the nonlinearity involves only the reduced probability density $\rho(\mathbf{q}, t)$ and does not involve a momentum variable $\mathbf{p}$. 


\section{APPENDIX D: LINEAR STABILITY ANALYSIS BASED ON EQ. (36): RIGOROUS PROOFS}

Following [18], Sec. 5.1.2, we prove that a perturbation $\tilde{\boldsymbol{\epsilon}}$ decreases or increases as a function of time by studying how appropriately defined norm measures $\|\tilde{\epsilon}\|$ evolve with time. To this end, let us first assume that the inequality $\kappa \rho_{\text {st }}(q)+Q>0$ holds for any $q$. In this case, we define the norm $\|\tilde{\epsilon}\|=\sqrt{\delta^{2} F_{q}\left[\rho_{\mathrm{st}}\right](\tilde{\epsilon})}$ that involves $\delta^{2} F_{q}$ as defined in Sec. II B 2 and reads explicitly

$$
\|\tilde{\epsilon}\|=\sqrt{\int\left[\kappa+\frac{Q}{\rho_{\mathrm{st}}(q)}\right] \tilde{\epsilon}^{2}(q) \mathrm{d} q .}
$$

Differentiating $\|\tilde{\epsilon}\|$ with respect to $\tau$, we obtain

$$
\frac{\mathrm{d}}{\mathrm{d} \tau}\|\tilde{\epsilon}\|=\frac{1}{\|\tilde{\epsilon}\|} \int\left[\kappa+\frac{Q}{\rho_{\mathrm{st}}(q)}\right] \tilde{\epsilon}(q, \tau) \frac{\partial}{\partial \tau} \tilde{\epsilon}(q, \tau) \mathrm{d} q .
$$

Substituting Eq. (36) into Eq. (D2) and using integration by parts, we obtain

$$
\frac{\mathrm{d}}{\mathrm{d} \tau}\|\tilde{\boldsymbol{\epsilon}}\|=-\frac{1}{\|\tilde{\epsilon}\|} \int \rho_{\mathrm{st}}(q)\left[\frac{\partial}{\partial q}\left(\kappa+\frac{Q}{\rho_{\mathrm{st}}(q)}\right) \tilde{\epsilon}(q, \tau)\right]^{2} \mathrm{~d} q \leq 0 .
$$

We see that the norm decreases - as indicated. Since the most right-standing equal sign in Eq. (D3) only holds for $\tilde{\boldsymbol{\epsilon}}=0$, we conclude that the square of the norm $\|\tilde{\epsilon}\|^{2}$ decreases strictly monotonically and converges to zero when the perturbation $\tilde{\boldsymbol{\epsilon}}$ converges to zero everywhere.

Next, let us assume that the inequality $\kappa \rho_{\text {st }}(q)+Q<0$ holds for any $q \in I$ with $I=[-c, c]$ and $c>0$. In this case, we study the evolution of a perturbation $\tilde{\epsilon}(q)$ which vanishes for $q \notin I$. That is, we require that $\tilde{\epsilon}(q)$ solves Eq. (36) under the boundary conditions $\tilde{\boldsymbol{\epsilon}}(q= \pm c)=0$. Then, we define the norm $\|\tilde{\boldsymbol{\epsilon}}\|=\sqrt{-\delta^{2} F_{q}\left[\rho_{\text {st }}\right](\tilde{\boldsymbol{\epsilon}})}$ (see also [18], Sec. 5.1.2) that reads explicitly

$$
\|\tilde{\epsilon}\|=\sqrt{-\int_{-c}^{c}\left[\kappa+\frac{Q}{\rho_{\mathrm{st}}(q)}\right] \tilde{\epsilon}^{2}(q) \mathrm{d} q .}
$$

Differentiating $\|\tilde{\epsilon}\|$ with respect to $\tau$, we obtain

$$
\frac{\mathrm{d}}{\mathrm{d} \tau}\|\tilde{\boldsymbol{\epsilon}}\|=-\frac{1}{\|\tilde{\boldsymbol{\epsilon}}\|} \int_{-c}^{c}\left[\kappa+\frac{Q}{\rho_{\mathrm{st}}(q)}\right] \tilde{\boldsymbol{\epsilon}}(q, \tau) \frac{\partial}{\partial \tau} \tilde{\boldsymbol{\epsilon}}(q, \tau) \mathrm{d} q .
$$

Substituting Eq. (36) into Eq. (D5) and using integration by parts, we obtain

$$
\frac{\mathrm{d}}{\mathrm{d} \tau}\|\tilde{\boldsymbol{\epsilon}}\|=\frac{1}{\|\tilde{\epsilon}\|} \int_{-c}^{c} \rho_{\mathrm{st}}(q)\left[\frac{\partial}{\partial q}\left(\kappa+\frac{Q}{\rho_{\mathrm{st}}(q)}\right) \tilde{\epsilon}(q, \tau)\right]^{2} \mathrm{~d} q \geq 0 .
$$

We see that the norm increases with time which indicates that the perturbation increases as a function of time.
[1] S. Heifets, Phys. Rev. E 54, 2889 (1996).

[2] G. V. Stupakov, B. N. Breizman, and M. S. Pekker, Phys. Rev. E 55, 5976 (1997).

[3] S. Heifets and B. Podobedov, Phys. Rev. ST Accel. Beams 2, 044402 (1999).

[4] S. Heifets, Phys. Rev. ST Accel. Beams 4, 044401 (2001).

[5] M. Venturini and R. Warnock, Phys. Rev. Lett. 89, 224802 (2002).

[6] S. Heifets, Phys. Rev. ST Accel. Beams 6, 080701 (2003).

[7] A. W. Chao, Physics of Collective Instabilities in High Energy Accelerators (John Wiley and Sons, New York, 1993).

[8] Y. Shobuda and K. Hirata, Phys. Rev. E 60, 2414 (1999).

[9] M. Venturini, Phys. Rev. ST Accel. Beams 5, 054403 (2002).

[10] H. Haken, Z. Phys. 263, 267 (1973).

[11] M. Hongler and D. M. Ryter, Z. Phys. B 31, 333 (1978).

[12] W. Ebeling and H. Engel-Herbert, Physica A (Amsterdam) 104, 378 (1980).

[13] W. Ebeling and L. Schimansky-Geier, in Noise in Nonlinear Dynamical Systems, Vol. 1, edited by F. Moss and P.V.E. McClintock (Cambridge University Press, Cambridge, England, 1989), pp. 279-306.

[14] F. Schweitzer, W. Ebeling, and B. Tilch, Phys. Rev. E 64, 021110 (2001).

[15] F. Schweitzer, Brownian Agents and Active Particles (Springer, Berlin, 2003).

[16] W. Ebeling and I. M. Sokolov, Statistical Thermodynamics and Stochastic Theory of Nonequilibrium Systems (World Scientific, Singapore, 2004).

[17] H. Risken, The Fokker-Planck Equation-Methods of Solution and Applications (Springer, Berlin, 1989).

[18] T.D. Frank, Nonlinear Fokker-Planck Equations: Fundamentals and Applications (Springer, Berlin, 2005).

[19] M. Shiino, Phys. Rev. A 36, 2393 (1987).

[20] M. Shiino, Phys. Rev. E 67, 056118 (2003).

[21] T. D. Frank, Phys. Lett. A 305, 150 (2002).

[22] H.C. Öttinger and M. Grmela, Phys. Rev. E 56, 6633 (1997).

[23] Y. Shobuda and K. Hirata, Part. Accel. 62, 165 (1999).

[24] T. D. Frank, Phys. Lett. A 319, 173 (2003).

[25] T. D. Frank, Phys. Lett. A 329, 475 (2004).

[26] T. D. Frank, Phys. Lett. A 337, 224 (2005).

[27] Y. Shobuda and K. Hirata, Phys. Rev. E 64, 067501 (2001).

[28] R.L. Warnock and J.A. Ellison, Phys. Rev. ST Accel. Beams 6, 104401 (2003).

[29] J. Haïssinski, Nuovo Cimento Soc. Ital. Fis. B 18, 72 (1973).

[30] C. Thomas, R. Bartonlini, J. I. M. Botman, G. Dattoli, L. Mezi, and M. Migliorati, Europhys. Lett. 60, 66 (2002).

[31] S. Petracca, T. Demma, and K. Hirata, Phys. Rev. ST Accel. Beams 8, 074401 (2005).

[32] E.W. Weisstein, CRC Concise Encyclopedia of Mathematics (Chapman and Hall/CRC, Boca Raton, 1998).

[33] S. Chandrasekhar, Rev. Mod. Phys. 15, 1 (1943). 